\title{
PO3-3-1
}

Poster session

\section{Apolipoprotein E deletion protects prostacyclin receptor agonist-induced relaxations in mouse aorta}

\author{
Yanhua Cheng, Paul M Vanhoutte, Susan Ws Leung \\ Pharmacology \& Pharmacy, The University of Hong Kong, Hong Kong
}

\section{Background:}

Nitric oxide-mediated, endothelium-dependent relaxations to acetylcholine of isolated arteries are blunted by ageing and high fat diet, as well as by apolipoprotein E deletion. The present study was designed to examine the effects of apolipoprotein E deletion on endothelium-dependent responses to prostacyclin (IP) receptor activation.

Methods:

Five-week old wild-type mice and apolipoprotein E-deficient (ApoE-/-) mice were fed with normal chow or high fat diet for 29 weeks. Aortae were isolated, contracted with phenylephrine and relaxed with increasing concentrations of iloprost (IP receptor agonist), and the isometric tension was measured in a Halpern-Mulvany myograph. Protein presences and cyclic nucleotide levels were measured by Western blotting and ELISA, respectively. Protein presences were also detected using immunofluorescence.

Results:

Iloprost, induced endothelium-, nitric oxide synthase-, and IP receptor-dependent relaxations in the aortae of young wildtype mice. High fat diet favored the activation of thromboxane-prostanoid (TP) receptors by iloprost causing contraction. Apolipoprotein E was present in the plasma and aortae of wild-type mice, especially in the endothelium; its presence was augmented by high-fat diet. Apolipoprotein E deletion potentiated the iloprost-induced relaxation in the aortae of young mice, and prevented the blunting of this response by high fat diet. The cAMP level was higher, but that of cGMP was lower in the aorta of ApoE-/- mice compared with same age wild-type mice. The protein presence of IP receptors was not different between the age-matched wild-type and ApoE-/- mice, but significantly reduced after high fat diet in ApoE-/mice.

Conclusions:

Iloprost induces an endothelium-dependent relaxation in the aorta of young healthy mice which involves both the cGMP and cAMP pathways; this response is blunted by prolonged exposure to a high fat diet. Apolipoprotein E deletion potentiates relaxations to IP receptor activation irrespective of age and diet. 